\title{
Improving gas distribution and evaporation in a gas cooling tower at Norcem Brevik
}

\author{
Fredrik Kasin*, Are Larsen, Christian Andersen, Rajan Jaiswal, Rajan K. Thapa \\ Department of Process, Energy and Environmental Technology, University of South-Eastern Norway, \\ fkasinehotmail.com, arelarsen@hotmail.com, christiana.1990@gmail.com \\ \{rajan.jaiswal, rajan.k.thapa\} @usn.no
}

\begin{abstract}
In this work the effect of the geometry of a GCT (Gas Cooling Tower) on the flue gas distribution in a cooling tower in Norcem, Brevik is investigated by using CPFD (Computational Particle Fluid Dynamics) modeling and simulation. Barracuda Virtual Reactor is used to perform the simulations of a baseline model, representative of the existing cooling tower. Results from simulations show poor distribution of flue gas and recirculation zones occurring on both sides of the distribution screen. The cause of this uneven distribution is attributed to challenging geometry and poor screen performance. To counteract this problem, a new model is developed with a second screen and guide vanes. The screens are placed in the lower part of the GCT's diffusor, whilst the guide vanes are in the diffusor's inlet duct. The screens and guide vanes are modeled as baffle computational cells with zero thickness. The implementation of a second screen eliminates the recirculation zone below the screens and improve the distribution.
\end{abstract}

Keywords: Norcem, Brevik, CPFD, cooling tower, gas distribution, Barracuda

\section{Introduction}

Concrete is the most used building material in the world. Norcem AS annually produces 1.7 million tons of cement at its two plants in Norway. The Brevik plant has always focused on technological innovation and has therefore been at the frontline of efficient and environmentally friendly cement production. Cement production is an energy-intensive process and reuse of waste heat is an important part of this. Waste heat from the preheater is used for drying raw materials, and the temperature must, therefore, be controlled. The temperature of the preheater flue gas is about 380$410^{\circ} \mathrm{C}$. The gas should have a temperature of about 190$290^{\circ} \mathrm{C}$ to use for drying of the raw materials and prevent damage to equipment. To control the temperature, the gas is cooled in a cooling tower by water spray. The cooling tower is designed as a cylinder, with a maximum diameter of $6 \mathrm{~m}$. Figure 1 shows the upper part of the cooling tower and a gas distribution screen. The gas is fed into the top of the tower and passes through a diffuser. In the lower part of the diffuser there is a distribution screen which has a thickness of $3 \mathrm{~mm}$ and $70 \mathrm{~mm}$ holes throughout the cross section. The aim of the screen is to distribute gas evenly throughout the cross- sectional area of the tower. The gas is cooled by cold water injected through 16 nozzles placed evenly around the perimeter of the tower at $1600 \mathrm{~mm}$ below the distribution screen. Each nozzle is placed $22.5^{\circ}$ apart from each other and they have pressure 25-27 bar.
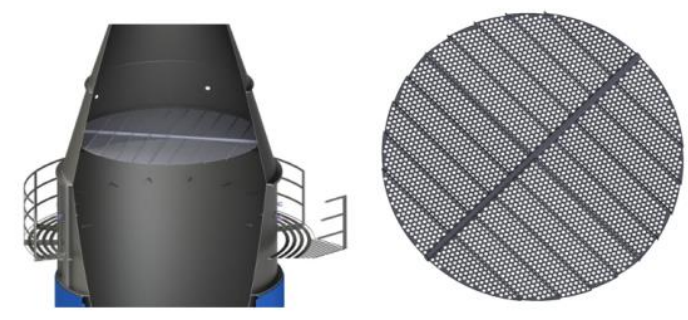

Figure 1. Upper part of cooling tower and gas distribution screen

Today's design makes use of only one gas distribution screen which results in insufficient distribution thus incomplete evaporation of cooling water. This creates spilling formation at the bottom of the cooling tower. This prevents deposition of dust and results in build ups and increased wear which leads to reduced production capacity. It is suspected that the screen is not able to distribute the gas evenly. Therefore, it is important to understand the actual conditions that causes the challenges and look at possible measures.

To investigate the causes that create the problems, data for geometry and flow parameters along the cooling tower are obtained from Norcem and analyzed to make a simulation model in CPFD software Barracuda VR. This will provide a basis for how the gas distribution can be improved. When using simulation tools, consideration is given to what kind of changes should be made and what effects this will have.

\section{Computational model}

In this work, a Computational Particle Fluid Dynamics (CPFD) model is used to calculate gas, particle, and water flow along the cooling tower. Gas and particles are passing through the distribution screen. All simulations are run in Barracuda Virtual Reactor 17. Barracuda VR uses the Multi-Phase Particle in Cell (MP-PIC) approach, where combined Eulerian and Lagrangian methods are used for the modeling of fluidsolid interaction. The fluid phase is solved as the continuum, while the particle phase is solved using the Lagrangian method. Langrangian method follows a 
particle independent of the grid, and its properties are calculated at each point/time step. In Eulerian, control volume method, the geometry is divided into several control volumes, and what goes in must go out. The number of control volumes are determined by the size of the grid used. The equations are solved at each point of the grid (Falkovich, 2011). The equations involved in the computational model presented in this section are proposed by O'Rourke et al. and Snider et al. and the detail about the governing equation and computational schemes can be found there (Andrews, 1996; Sinder, 2001).

The fluid phase is described with Navier-Stokes mass, impulse, and energy equation (Thapa, 2015). They describe how the pressure, velocity, temperature, viscosity, and density of a moving gas are related. The equations contain several dependent variables, such as velocity and viscosity, as a function of four independent variables $\mathrm{x}, \mathrm{y}, \mathrm{z}$, and $\mathrm{t}$. The equations are thus partial differential equations (Hall, 2015)

One of the most important parameters in CPFD is the drag model. The drag model indicates forces acting on a particle from a fluid. In simulations, it is chosen to use Gidaspow's model for both particles and water. This model is based on Wen \& Yu and Ergun's models. Wen $\&$ Yu's model is adapted to flows with low particle fractions. Ergun's model is adapted to large volume fractions and will not be used in this work.

\subsection{Model parameter}

The composition of the flue gas coming into the cooling tower consists of several fractions, but the main constituents are nitrogen, carbon dioxide, oxygen, water vapor, and carbon monoxide. The volume fraction of these will vary with the type of clinker produced and how the process is operated. What types of fuel and quantities of these will be important factors. The composition used in this report is from the production of Standard clinker and measured in connection with a heat balance performed in 2018 and is shown in Table .

Table 1. Composition of flue gas

\begin{tabular}{|l|c|c|}
\hline Component & $\begin{array}{c}\text { Volume } \\
\text { fraction }\end{array}$ & $\begin{array}{c}\text { Molecular } \\
\text { weight }[\mathrm{g} / \mathrm{mol}]\end{array}$ \\
\hline $\mathrm{N}_{2}$ & $62.7 \%$ & 28.0134 \\
\hline $\mathrm{CO}_{2}$ & $23.5 \%$ & 44.0095 \\
\hline $\mathrm{H}_{2} \mathrm{O}$ & $9 \%$ & 18.0152 \\
\hline $\mathrm{O}_{2}$ & $4.6 \%$ & 31.9988 \\
\hline $\mathrm{CO}$ & $2 \%$ & 28.0101 \\
\hline
\end{tabular}

The models include data on the particles that are part of the simulation. This includes particle type, size distribution, sphericity, and particle density. Raw meal has been used as a starting point for particle parameters, although this will be a mixture of raw meal, calcined raw meal, and ash residue. The size distribution for solid particles is an average distribution over a year of production of Standard raw meal. The particle size distribution (PSD) used in the computational model is shown in Figure 2.

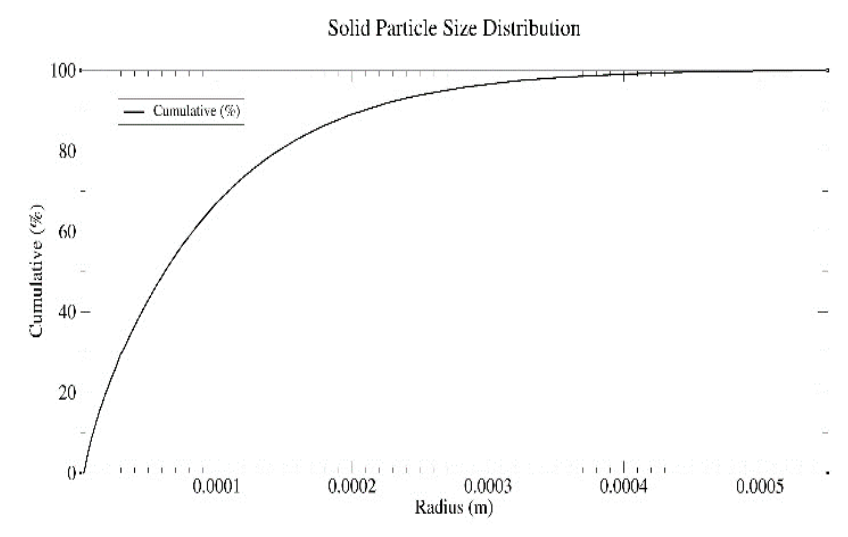

Figure 2. Solid particle size distribution, for raw meal For the initial simulations only water droplets are added to the model. The droplet size distribution is shown in Figure 3.

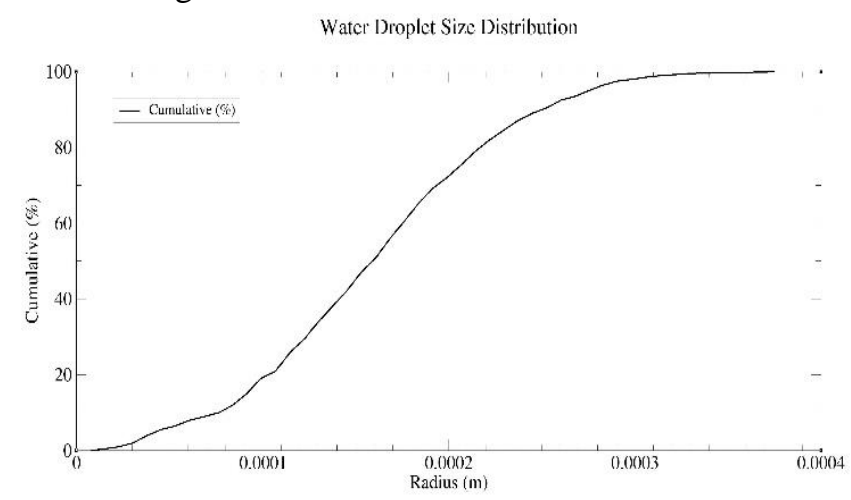

Figure 3. Water droplet size distribution

The droplet size effects the overall performance of the cooling tower. Fine droplets accelerate the evaporation process and hiders transportation of moisture out of the tower.

\subsection{Model geometry and boundary conditions}

A model of geometry is prepared for the cooling tower. To model the cooling tower on the correct scale and with the necessary level of detail, production drawings of the inlet duct, cooling tower, and outlet provided by Norcem have been used. The drawings are hand drawn from the period 1978 to 1986 . The drawings are the starting point for the model. Some of the parameters were missing and new physical measurements have been made to find out the missing geometry parameters. This applies mainly to the lower part of the tower with piles and outlet duct. New measurements have yielded a margin of error against original drawings by $0.07 \%$. 
CPFD software Barracuda reads only massive parts of the model as a flow path. The tower is thus modeled as a massive model where nozzle pipes are modeled as cavities. The boundary to the surroundings is then determined by the inner diameter of the tower mantle.

The model is drawn according to the right-hand rule so that the $\mathrm{z}$-axis is the vertical axis in the model. To make sure this is correct, the model is drawn in a positive $\mathrm{z}$ axis. This is done so that the axis representing the gravitational direction should correspond between the two programs. The only simplification of the model that has been done is to omit the screw conveyor at the bottom as it will not significantly affect the flow. The geometry was drawn in CAD software SolidWorks, saved as an STL file, and then imported to Barracuda. The model geometry of the tower and boundary conditions are shown in Figure 4..
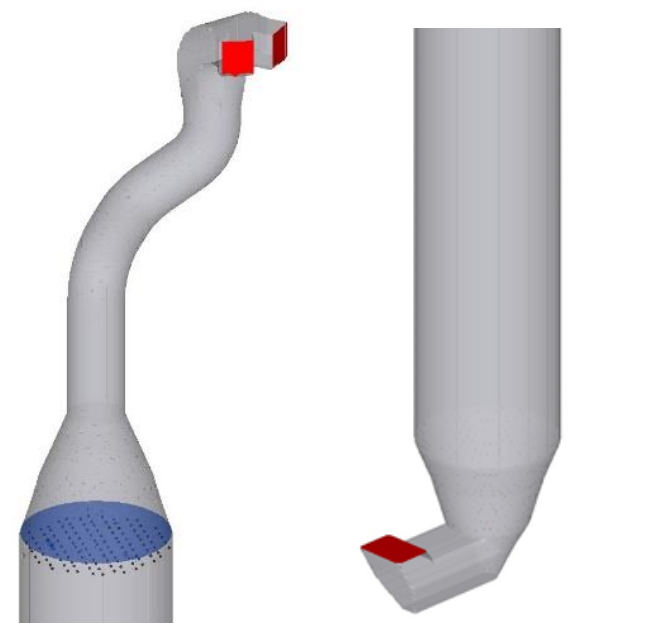

Figure 4. Model geometry and boundary conditions

For the isothermal gas flow simulations both inlet and outlet of the cooling tower are defined as flow boundary. This is because the inflow and outflow are set equal. When injection of water is added to the simulation the outlet boundary is changed to pressure BC. This defines outlet conditions based on pressure. Injection boundaries are used for water nozzles.

\section{Simulation methods}

A series of simulations were run using the baseline model. Before simulations, physical monitors were installed under and above the distribution screen and the pressure drop was measured to $70 \mathrm{~Pa}$. This measurement is used in the CPFD model.

The main objective of this work is to improve the gas distribution so that better evaporation of the cooling water can be achieved. To measure an improvement, a list of criteria has been set for velocity differences over the tower cross-section:

- Measurements must be made across the entire cross-section, except for a peripheral area behind nozzles. This area should not exceed $10 \%$ of the total cross-section.

- Horizontal distance between the measuring points should not exceed $500 \mathrm{~mm}$.

- Recirculation zones must not occur within the evaporation zone.

- The velocity deviations in the measuring points should not exceed $+/-30 \%$ of the calculated velocity for a given cross-section. This gives a permissible deviation from -2.26 to $-4.12 \mathrm{~m} / \mathrm{s}$.

- Speed deviations in the peripheral zone must not exceed $+/-50 \%$ of the calculated speed.

- The standard deviation for all measurement points should not exceed $18 \%$.

\section{Results and discussion}

\subsection{Baseline model with one distribution screen}

The first simulations were run for the entire tower without dust particle loading and water injection. The objective was to get an overview on how the gas flows in the area above and under the distribution screen where water nozzles are located. It is assumed that the volume fraction of dust is small enough that it does not significantly affect the gas flow. The simulation is set up with inlet and outlet Flow BC with equal mass flow and temperature. The boundary conditions for this model are shown in Table 2.

Table 2. Flow boundary conditions

\begin{tabular}{|l|c|c|c|}
\hline & $\begin{array}{c}\text { Pressure } \\
{[\mathrm{Pa}]}\end{array}$ & $\begin{array}{c}\text { Temperature } \\
{[\mathrm{K}]}\end{array}$ & $\begin{array}{c}\text { Mass flow } \\
{[\mathrm{kg} / \mathrm{s}]}\end{array}$ \\
\hline Inlet BC & 92073 & 673.15 & 52.15 \\
\hline Outlet BC & 91750 & 673.15 & -52.15 \\
\hline
\end{tabular}

In the cooling tower, the temperature variation, during normal operating conditions, does not exceed 12 degree Celsius. Therefore, the temperature at inlet and outlet boundaries are considered as constant.

The geometry of the model is divided into one million uniform cells as shown in Figure 5. which gives a cell size of $17 \mathrm{~cm}$. The grid size was not possible to reduce further because of very large computational time. If the grid were reduced further, it would have taken few weeks for one simulation which is the beyond of the scope of this work. 


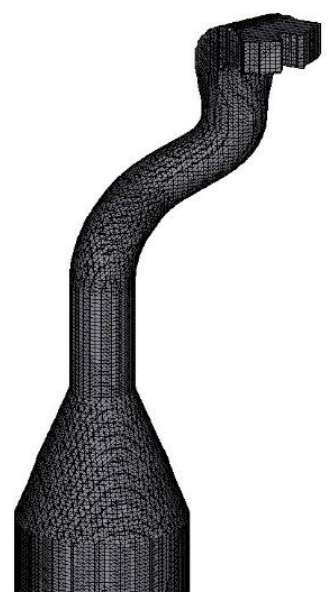

Figure 5. Grid of the geometry

The distribution screen is modeled similarly to a porous plate with a calculated resistance. In such a plate, particles will not be able to pass, and we only get a picture on how the gas flows. This is done as a simplification to save simulation time. For the current model, the simulation time is 12 hours. A modeling of the distribution screen with $3 \mathrm{~mm}$ thickness would have increased the need for cells to several billion and given a simulation time that is far from the possibilities of this work.

Results from this model show large velocity variations over the tower cross-section. Figure 6. shows the axial velocity in the XY plane at the level of water nozzles and the central axial velocity in the $\mathrm{YZ}$ plane.

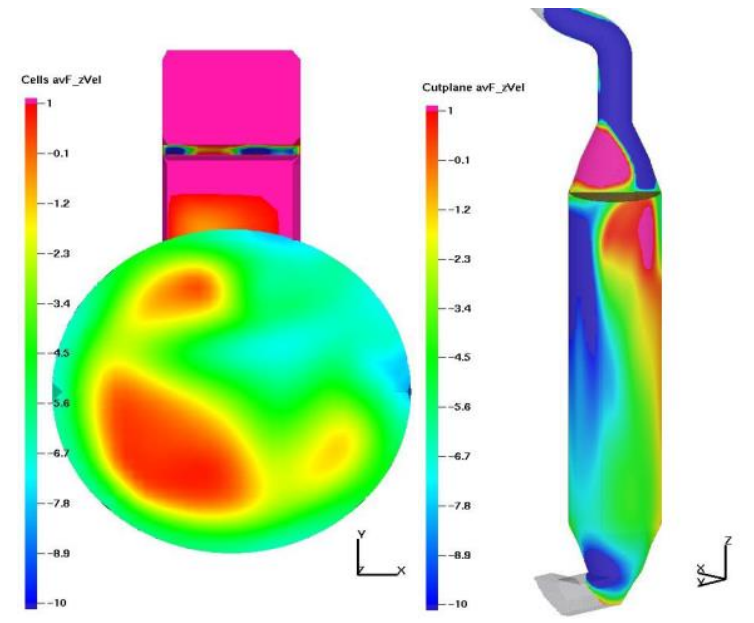

Figure 6. Velocity profile for the baseline model, one screen

From Figure 6., a clear distortion is seen under the distribution screen, where the gas flow changes the direction. The radial velocities increase in the negative $\mathrm{x}$-direction up to the wall of the diffuser. Here the direction of flow changes and the axial velocity becomes positive. This is due to the geometry of the duct from the upper cyclone stage down to the diffuser. After the last duct bend, wall separation occurs, and the gas is pressed against one side, which continues all the way down to the distribution screen. Here, a recirculation zone is formed which further amplifies the effect of the skewed gas distribution. Consequently, the gas velocity through the screen becomes uneven.

As a consequence, a large recirculation area and stagnant gas pockets under the distribution screen. In these areas, evaporation of cooling water will be greatly reduced. The radial velocity components along the $\mathrm{x}$ and $y$ axes exceed the axial velocity in several areas. This will transport cooling water and particles to the tower walls where they agglomerate (Nielsen, 2002).

The simulation results from baseline model with one distribution screen show the following:

- The standard deviation of the velocity profile at the level of the water jets is $65.13 \%$

- For single measurements, the velocity ranges from 0.5 to $-7.05 \mathrm{~m} / \mathrm{s}$

- The average of each measurement point shows 50 measurements where the velocity is higher than $-4.21 \mathrm{~m} / \mathrm{s}$

- 34 measuring point has an average value where the velocity is lower than $-2.26 \mathrm{~m} / \mathrm{s}$

- A total of 84 of 119 measurements are outside the recommended range.

The pressure loss from the tower inlet to below the screen is $269 \mathrm{~Pa}$.

\subsection{Model with two distribution screens}

In the next model a second distribution screen has been added $1.95 \mathrm{~m}$ above the original one. The results from this simulation show a significant improvement of the gas distribution. Recirculation zones and stagnant gas pockets are eliminated, and the radial velocities are greatly reduced. Figure 7 . shows the axial velocity in the XY plane at the level of water nozzles and the central axial velocity in the $\mathrm{YZ}$ plane.

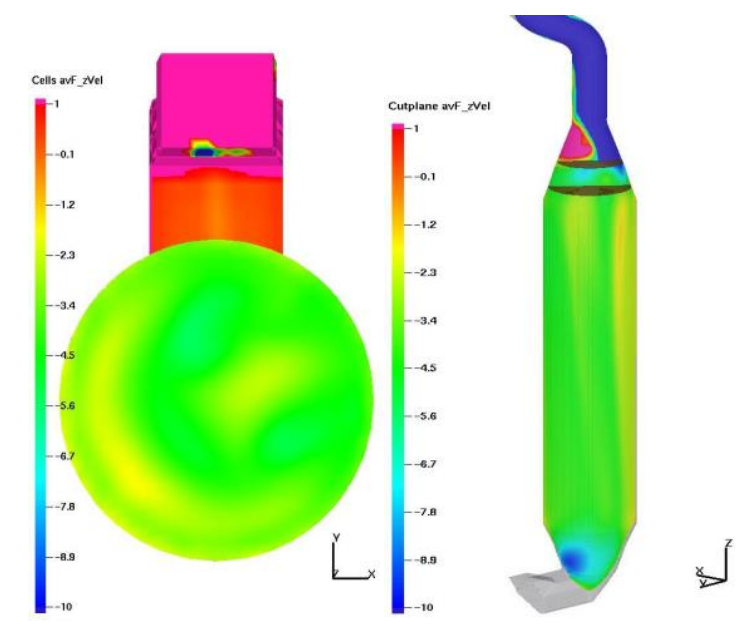

Figure 7. Velocity profile for model with two screens

The major results from the second model summarized:

- The standard deviation of the velocity profile at the level of water jets is $21 \%$

- The velocity ranges from -1.75 to $-5.52 \mathrm{~m} / \mathrm{s}$ 
- A total of 43 of 119 measurements are outside the recommended range.

The pressure loss for this model is $278 \mathrm{~Pa}$, an increase of 9Pa from the baseline model.

\subsection{Models with water injection}

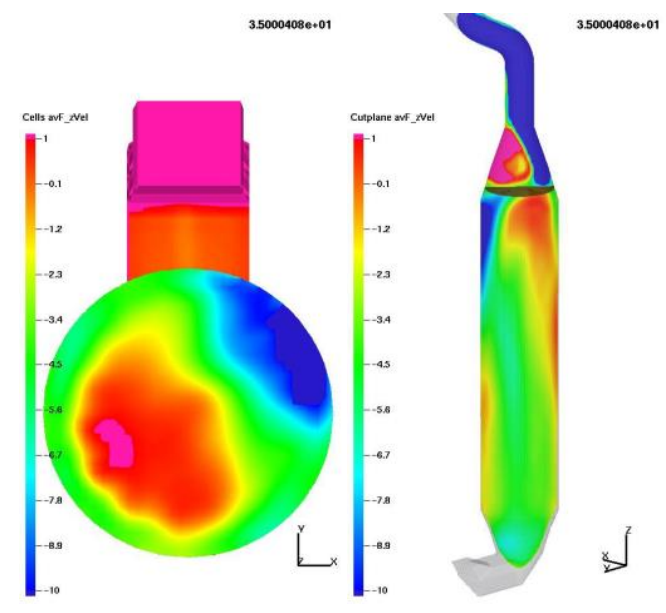

Figure 8. Velocity profile with water injection, one screen

Based on the baseline and second model, water injection was added to see how this affects the gas distribution and pressure loss. The model is set up with inlet Flow BC and outlet Pressure BC. Outlet pressure is specified to represent the measured value. Water is injected through an Injection $\mathrm{BC}$ via 16 simplex spillback atomizers placed $500 \mathrm{~mm}$ below the diffuser at the rate of $3.74 \mathrm{~kg} / \mathrm{s}$. For the baseline model an increased velocity deviation and strengthening of recirculation zone is seen (see Figure 6.). The recirculation zone creates a zone of stagnant gas along the tower wall where water spray and particles will agglomerate and cause corrosion. This area correlates with tower wall corrosion seen over the past few years. Opposite to the recirculation zone, a high velocity zone is created where complete evaporation is not achieved within the tower. The pressure loss from inlet to below screen is $858 \mathrm{~Pa}$.

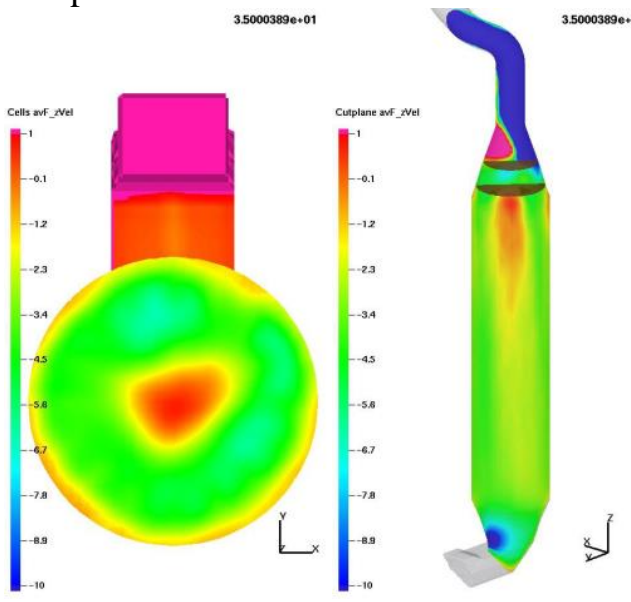

Figure 9. Velocity profile with water injection, two screens

For the model with two screens, see Figure 9. a recirculation zone is created centrally in the tower. The following inward radial velocities prevents the transportation of particles to the tower walls. This model shows almost complete evaporation of water droplets before flue gas exits tower. By using flux planes this amount is calculated to $6.6 \mathrm{~kg} / \mathrm{min}$ at tower exit. The pressure loss from inlet to below screens is $859 \mathrm{~Pa}$. Thus, the impact of a second screen on gas transportation is negligible.

The results show that the main contributor to pressure loss is water injection and not the distribution screen(s). This occurs despite a lower outlet gas velocity.

\subsection{Models with new nozzles}

Simulations were further run for the tower with new nozzle inclined at $45^{\circ}$ with spray angle of $60^{\circ}$. The droplet size distributions are kept similar to the prior simulations. All other parameters are also kept unchanged. The first simulation were run with one distribution screen. The results show more water and particle accumulation near the wall of the tower which will negatively effect on evaporation process and gas distribution.

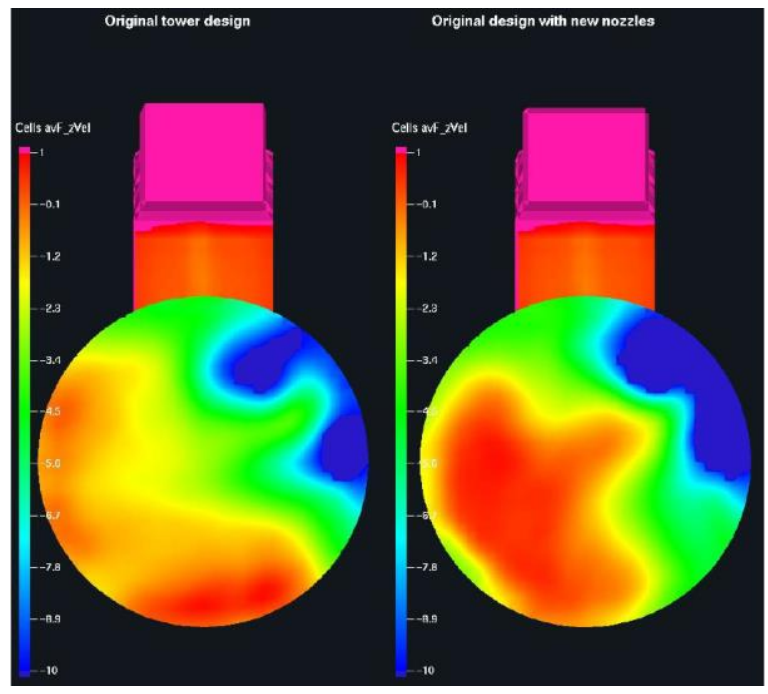

Figure 10. Comparison of gas velocities at $2500 \mathrm{~mm}$ below the distribution plate

Simulations were also run for the tower with two distribution screens and new nozzles. The gas velocity distribution along the tower is presented in Figure 11.

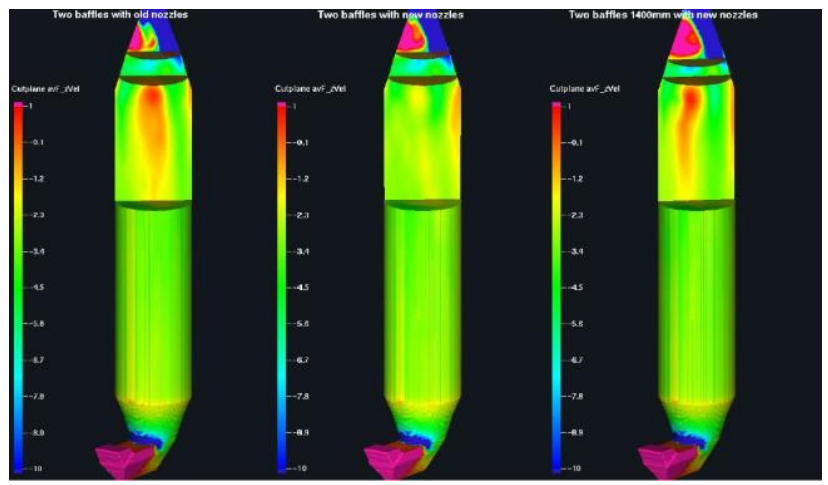

Figure 11. Comparison of gas velocities along the center of the tower 
Figure shows that the gas distribution at the tower with new nozzles and two distribution screens is improved.

\section{Conclusions}

Results from simulations done on the second model with two distribution screens show an improved gas distribution and complete evaporation of cooling water. This is achieved without significant increase in the pressure loss through the tower. Nevertheless, some recirculation occurs along the walls in the upper part of the evaporation zone. This is due to wall separation below the lowermost distribution screen. Small radial velocities that occur may transport particles to tower walls in this area. The gas distribution in the tower with two distribution screen and new nozzles gives better results.

\section{References}

M. J. Andrews and P. J. O'Rourke. The multiphase particlein-cell (MP-PIC) method for dense particulate flows, International Journal of Multiphase Flow, 22(2): 379-402, 1996.

G. Falkovich. Fluid Mechanics: A Short Course for Physicists. Cambridge University Press, 2011.

N. Hall. Navier-Stokes Equations. NASA, 05.2015 https://www.grc.nasa.gov/WWW/k12/airplane/nseqs.html

N. F. Nielsen and L. Lind. Applying CFD for Design of Gas Conditioning Towers with Swirling Flow, presented at the ASME 2002 Pressure Vessels and Piping Conference, Vancouver, 2002. https://doi.org/10.1115/PVP2002-1588

D.M. Snider, P.J. O'Rourke, and M.J. Andrews. An incompressible three-dimensional multiphase particle-incell model for dense particle flows, Journal of Computational Physics, 170(2): 523-549, 2001.

R. K. Thapa, A. Frohner, G. Tondl, C. Pfeifer, and B. M. Halvorsen. Circulating fluidized bed combustion reactor: Computational Particle Fluid Dynamic model validation and gas feed position optimization, 2015 DOI: https://www.sciencedirect.com/science/article/pii/S0 098135416301661. 